\title{
OAB-Therapie mit Trospiumchlorid-Retard wirksam und verträglich
}

- Trospiumchlorid - der Klassiker unter den Antimuskarinika - zählt zu den am häufigsten verschriebenen Substanzen für Patienten mit überaktiver Blase (OAB). Der Wirkstoff ist eine relativ große, polare und wenig lipophile Ammoniumverbindung. Diese Eigenschaften sorgen dafür, dass das Molekül die Blut-HirnSchranke kaum überwinden kann. Deshalb sei das Risiko für zentralnervöse Nebenwirkungen sehr gering, so Dr. Christian Gratzke aus München. Ein weiterer Vorteil der Substanz ist, dass sie bis zu $80 \%$ unverändert renal ausgeschieden wird: Das Risiko für metabolische Medikamenteninteraktionen ist daher gering.

In der Retardformulierung von Trospiumchlorid (uriVesc ${ }^{\oplus}, 60 \mathrm{mg}$ einmal täglich) sind Wirksamkeit und Verträglichkeit im Vergleich zu bisherigen nicht modifizierten
Freisetzungsformen noch verbessert worden. Dies konnten zwei placebokontrollierte randomisierte Phase-III-Studien mit insgesamt 1.165 OAB-Patienten zeigen. Bereits nach einwöchiger Therapie mit Trospiumchlorid-Retard hatten sich in den Verumgruppen die Miktionshäufigkeit und die täglichen Dranginkontinenzepisoden im Vergleich zu Placebo signifikant verringert. Nach drei Monaten hatten die Dranginkontinenzepisoden schließlich um $80 \%$ (vs. 56\% unter Placebo) abgenommen. Signifikante Therapieeffekte wurden zudem bei älteren Patienten ab 75 Jahren und bei Adipösen nachgewiesen.

Als häufigste Nebenwirkung trat Mundtrockenheit auf. Im Vergleich zu anderen Studien mit nicht modifizierter Freisetzung von Trospiumchlorid trat dies bei der Retard-
Formulierung allerdings nur halb so häufig auf. Auch mit den lange Zeit unbeachteten ZNS-Nebenwirkungen unter Anticholinergika - beispielsweise Konzentrations- und Gedächtnisstörungen - ist bei einer Therapie mit Trospiumchlorid kaum zu rechnen. Die Substanz ist nicht liquorgängig, solange die Blut-Hirn-Schranke intakt ist.

In der PRISCUS-Liste - einer Auflistung von Medikamenten, die bei älteren Patienten nicht eingesetzt werden sollten - ist Trospiumchlorid als einziger Vertreter von urologischen Spasmolytika als empfehlenswert eingestuft worden.

Nach Informationen von Rottapharm I Madaus, Köln

\section{Neues Testosteron-Gel mit höherer Wirkstoffkonzentration}

— Mit Testotop ${ }^{\circledR}$ Gel in den Stärken 62,5 mg und $125 \mathrm{mg}$ führt das Unternehmen Galenpharma neue Produkte zur topischen Testosteronsubstitution des Hypogonadismus ein. Testotop ${ }^{\circledR}$ Gel zeichnet sich insbesondere durch den Testosterongehalt von 2,5\% aus, der es ermöglicht, die therapeutisch erfor- derliche Wirkstoffmenge in einer deutlich geringeren Gelmenge als bislang üblich zu applizieren.

Mit einem Testosterongehalt von 2,5\% ist Testotop ${ }^{\circledR}$ Gel derzeit das am höchsten konzentrierte topische Testosteronpräparat auf dem Markt. Die dadurch mögliche

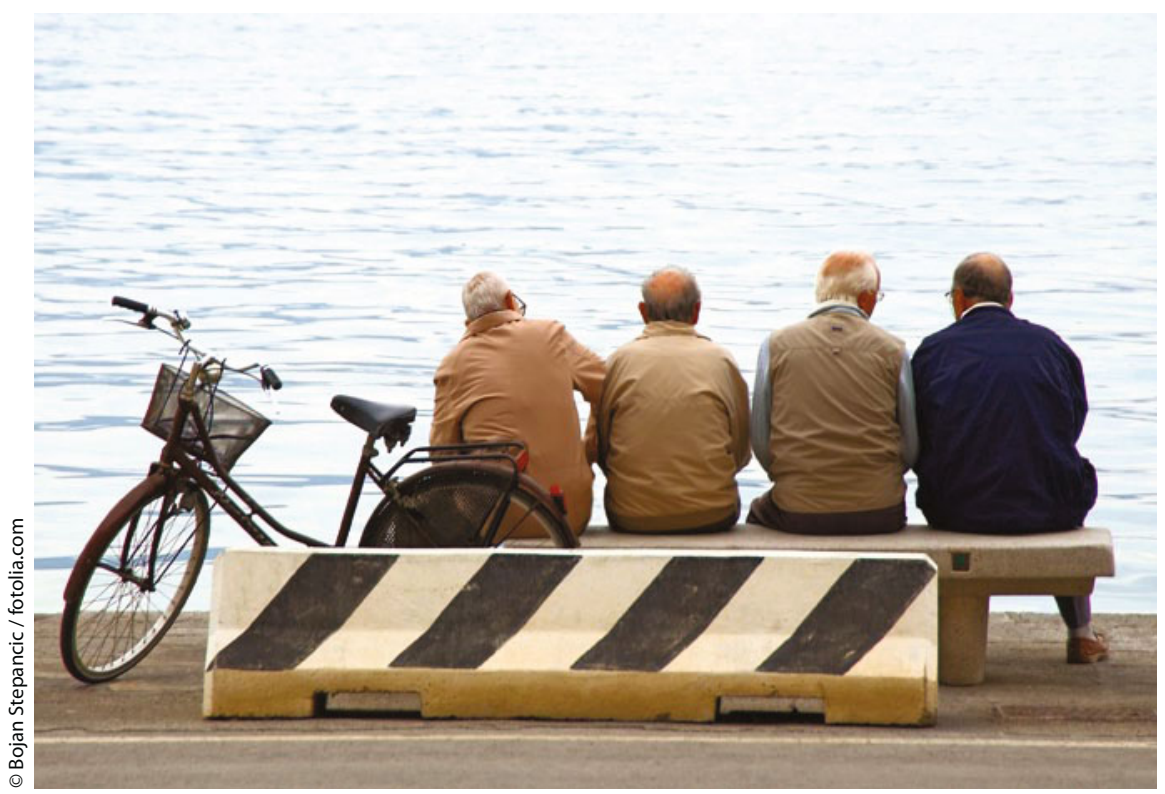

Testosteronsubstitution für Männer mit unterschiedlich stark erniedrigten Testosteronspiegeln. deutliche Verringerung der zu applizierenden Gelmenge ist geeignet, das bislang von Urologen und Endokrinologen häufig beobachtete Compliance-Problem bei der Auftragung größerer Gelmengen zu beseitigen. An Männern mit niedrigen Ausgangswerten von $2-3 \mathrm{nmol} / \mathrm{l}(0,58-0,87 \mathrm{ng} / \mathrm{ml})$ wurde gezeigt, dass bereits nach fünf Tagen Anwendung der 125- mg-Stärke physiologische Testosteronspiegel erreicht werden können [Rolf $C$ et al. Eur J Endocrinol 2002; 146: 673-9]. Ferner konnte nachgewiesen werden, dass das Gel bereits zehn Minuten nach dem Auftragen wieder abgewaschen werden kann, ohne dass die Hormonresorption hierdurch negativ beeinflusst wird. Die Möglichkeit, Testotop ${ }^{\circledast}$ Gel nach so kurzer Zeit wieder abzuwaschen, verbessert den Komfort des Patienten und erhöht die Sicherheit durch Verringerung des Übertragungsrisikos auf Kontaktpersonen.

Testotop ${ }^{\circledR}$ Gel ist zur Testosteronsubstitution des männlichen Hypogonadismus zugelassen. Es ermöglicht die Behandlung von Männern mit unterschiedlich stark erniedrigten Testosteronspiegeln.

Nach Informationen von Galenpharma, Kiel 